\title{
Soya intake and plasma concentrations of daidzein and genistein: validity of dietary assessment among eighty British women (Oxford arm of the European Prospective Investigation into Cancer and Nutrition)
}

\author{
Pia K. Verkasalo ${ }^{1 *}$, Paul N. Appleby ${ }^{1}$, Naomi E. Allen ${ }^{1}$, Gwyneth Davey $^{1}$, \\ Herman Adlercreutz ${ }^{2,3}$ and Timothy J. Key ${ }^{1}$ \\ ${ }^{1}$ Imperial Cancer Research Fund, Cancer Epidemiology Unit, University of Oxford, Oxford, UK \\ ${ }^{2}$ Institute for Preventive Medicine, Nutrition and Cancer, the Folkhälsan Research Center, Helsinki, Finland \\ ${ }^{3}$ Department of Clinical Chemistry, University of Helsinki, Helsinki, Finland
}

(Received 12 December 2000 - Revised 1 May 2001 - Accepted 10 May 2001)

\begin{abstract}
Soya products contain high levels of the isoflavones genistein and daidzein, and their glucosides, and may lower the risk of cardiovascular disease, osteoporosis and cancer. The present crosssectional study investigated plasma concentrations of daidzein and genistein and their correlations with dietary soya consumption in four groups of twenty premenopausal British women. The women were selected from the Oxford arm of the European Prospective Investigation into Cancer and Nutrition using data from food-frequency questionnaires (FFQ) to guarantee a wide variation in soya consumption, and to investigate the utility of the question related to soya milk consumption compared with the utility of the question related to other soya foods. Dietary intakes of isoflavones were additionally assessed by $7 \mathrm{~d}$ food diaries. Plasma concentrations of daidzein and genistein were measured by time-resolved fluoroimmunoassay. Geometric mean plasma concentrations (nmol/l) were for the four groups, which were based on increasing soya intake, 4.9, 8.4, 39.2 and 132 for daidzein and 14.3, 16.5, 119 and 378 for genistein. The Spearman correlation coefficients for plasma isoflavone concentrations with estimated dietary intakes were between 0.66 and 0.80 for the diary-based estimates and between 0.24 and 0.74 for the FFQ-based estimates. The correlations for soya milk intakes were clearly higher than the correlations for intakes of other soya foods. We conclude that both the food diary and the FFQ estimate dietary soya isoflavone intakes sufficiently well to use them in epidemiological studies, and that plasma concentrations of daidzein and genistein in Western women who consumed soya products as a part of their regular diet were close to those in Asian populations.
\end{abstract}

Diet records: Isoflavones in blood: Questionnaires: Soyabeans

The isoflavones genistein and daidzein, and their glucosides, are naturally-occurring hormone-like substances that are present at high levels in soyabeans. It has been proposed that isoflavones may lower the risks of cardiovascular disease, osteoporosis and cancer (Adlercreutz \& Mazur, 1997; Bingham et al. 1998; Fournier et al. 1998; Setchell, 1998). Ecological comparisons have shown that both breast cancer and prostate cancer are much rarer in populations in Japan and China, where soyabean consumption is high, than in Western countries, where soyabean consumption is low (International Agency for Research on Cancer, 2000). A few epidemiological studies have investigated the association between soya food consumption and breast cancer risk, but the results so far are inconsistent (Key et al. 1999). In Asian populations, high soyabean consumption is probably a marker of adherence to a traditional dietary pattern, and is likely to be correlated with other nutritional factors. It is, therefore, of considerable interest to study soya food consumption in Western populations, where the possible correlations with other nutritional factors are likely to be different. However, there are few studies of this type and soya food consumption tends to be low (Greenstein et al. 1996; Jacobsen et al. 1998).

The Oxford arm of the European Prospective Investigation into Cancer and Nutrition (EPIC-Oxford) includes

\footnotetext{
Abbreviations: EPIC-Oxford, Oxford arm of the European Prospective Investigation into Cancer and Nutrition; FFQ, food-frequency questionnaire.

* Corresponding author: Dr Pia K. Verkasalo, fax +44 1865310 545, email p.verkasalo@icrf.icnet.uk
} 
information on 57700 British subjects with a wide range of dietary habits, soyabeans being an important protein source among the large vegetarian $(n$ 16 000) and vegan $(n$ 2100) subpopulations. The aim of the present study was to investigate plasma concentrations of daidzein and genistein and their associations with dietary soya consumption in four groups of twenty premenopausal British women in the EPIC-Oxford cohort. In addition, we studied the utility of the questions related to soya milk consumption compared with the utility of the question related to consumption of other soya products.

\section{Materials and methods}

The EPIC-Oxford cohort consists of 57700 subjects (including 44500 women) aged 20 years and above and living in the UK between 1993 and 1999. The protocol of recruitment was approved by the Central Oxford Research Ethics Committee and by local ethics committees. Participants were recruited through collaborating general practitioners, vegetarian and vegan societies, vegetarian and health food magazines, or were friends or relatives of other participants. All participants completed an extensive foodfrequency questionnaire (FFQ; Bingham et al. 1997), which included three closed questions relating to soya intake. Two questions asked about the type of milk the subjects used most often and the amount used, including milk with tea, coffee, and cereals (none; 0.25 pint $(142 \mathrm{ml}) ; 0.5$ pint $(284 \mathrm{ml}) ; 0.75$ pint $(426 \mathrm{ml}) ; 1$ pint $(568 \mathrm{ml}) ;>1$ pint $)$. The daily consumption of soya milk $(\mathrm{g})$ was calculated based on the answers to these questions. The third question asked about the frequency of the use of soya foods such as tofu, soya meat, textured vegetable protein and vegeburgers during the last 12 months (never or less than once per month; one to three times per month; once per week; two to four times per week; five to six times per week; once per d; two to three times per d; four to five times per d; six or more times per $\mathrm{d}$ ); for convenience, these other soya foods will in the present paper be referred to as 'solid soya foods'.

On a separate occasion the subjects completed a $7 \mathrm{~d}$ food diary, which is an open-ended record of all food and drink consumed over this period. A previous validation study has indicated that the $7 \mathrm{~d}$ food diary estimates the intake of most nutrients more accurately than the FFQ; for example, the unadjusted and energy-adjusted correlation coefficients between $24 \mathrm{~h}$ urine excretion and dietary intake of $\mathrm{N}$ from $7 \mathrm{~d}$ food diaries were 0.65 and 0.67 respectively, the corresponding correlation coefficients for the FFQ being 0.24 and 0.49 (Bingham et al. 1997). Subjects were also asked to provide a $30 \mathrm{ml}$ blood sample, which was sent by mail to the central laboratory where plasma was prepared and stored for up to 6 months at $-70^{\circ} \mathrm{C}$ and then in liquid $\mathrm{N}_{2}$ until the samples were assayed in 1999.

The present study is based on a sample of eighty women, aged between 20 and 39 years, who were allocated to one of four groups of twenty women according to their consumption of soya foods estimated from the FFQ. Twenty women consumed no soya milk and very little solid soya food (once per month or less; group 1), another twenty consumed no soya milk but ate solid soya foods regularly (i.e. at least five to six times per week; group 2), twenty drank 0.25 pint
(142 ml) of soya milk daily and ate some of the solid soya foods (i.e. at most once per week; group 3) and twenty drank 0.5 pint $(284 \mathrm{ml})$ or more of soya milk daily and consumed solid soya foods at least two to four times per week (group 4). Group 1 was selected to include subjects with presumably low isoflavone intakes, groups 2 and 3 to include subjects with intermediate isoflavone intakes from two different sources and group 4 to include subjects with high isoflavone intakes. The intention was that group 2 would provide information on the validity of the FFQ question relating to other soya food consumption and that group 3 would provide information related to the validity of the FFQ questions on soya milk consumption. Before conducting the study, we estimated from the FFQ that the daily isoflavone intakes (mg) for groups $1-4$ would be $<1$, $18.4-23 \cdot 4,14 \cdot 2-37 \cdot 6$, and $\geq 38.4$ respectively; i.e. our expectation was that groups 2 and 3 would have overlapping isoflavone intakes but acquire them from two different major sources. Women were excluded from the study if they were on antibiotics or suffering from a chronic disease at the time of FFQ or venepuncture. For the women selected, the average time from FFQ to venepuncture was 5 months exactly, and the average time from venepuncture to completion of the food diary was 4 months and $10 \mathrm{~d}$.

Isoflavone intakes were calculated by multiplying the frequency of soya food consumption by portion weights to obtain g soya food consumed/d (for FFQ, a standard portion weight of solid soya foods was estimated to consist of $50 \mathrm{~g}$ tofu, $33 \mathrm{~g}$ vegeburger and $6 \mathrm{~g}$ textured vegetable protein). These were then converted to dietary intakes of daidzein, genistein and total isoflavones (including daidzein, genistein and glycitein) using measurements for daidzein, genistein and total isoflavones obtained from the US Department of Agriculture-Iowa State University (1999) database on the isoflavone content of foods. This database incorporates data on the isoflavone content of foods collected from scientific articles published in refereed journals, and by extensive sampling of soya-containing foods and subsequent laboratory analysis. The values for glucoside forms had been converted into free forms (aglycones) by using appropriate ratios of molecular weights, and then added to their respective free-form values to generate total values for daidzein and genistein.

Plasma concentrations of daidzein and genistein were measured by time-resolved fluoroimmunoassay at the Institute for Preventive Medicine, Nutrition and Cancer, the Folkhälsan Research Center, Helsinki, Finland (Wang et al. 2000). In a previous study the correlation coefficients between time-resolved fluoroimmunoassay and GLC-MS (the reference method) were very high ( $r$ values $0.95-$ 0.99, depending on concentration), indicating that this relatively simple and rapid assay provides valid measures of plasma isoflavones (Wang et al. 2000). In the present study the intra-assay percentage $\mathrm{CVs}$ at three different concentrations were $15 \cdot 7,8.7$ and 9.2 for daidzein and $44.5,4.9$ and 11.0 for genistein. The corresponding mean concentrations (nmol/1) were 1.96, 8.41 and 103 for daidzein and 2.18, 25.5 and 162 for genistein. It should be noted that the high intraassay $\mathrm{CV}$ at the lowest concentration of genistein is for a concentration much lower than the mean value obtained in the lowest-soya-intake group. 
The distributions of plasma isoflavone concentrations were positively skewed, and they were logarithmically transformed to produce approximately normal distributions. ANOVA models provided the estimates of means and $95 \%$ CI for the log-transformed plasma concentrations by soyaintake group and other measures of dietary soya intake; we exponentiated the latter values to obtain the geometric means and respective $95 \% \mathrm{CI}$. Arithmetic means are also shown to facilitate comparison with the published results of soya supplementation studies. Associations between plasma concentrations and dietary intake measures were investigated using Spearman correlation coefficients.

The blood samples had been in the mail on average 1.5 S.D. 1.0) d and, consequently, the average haemoglobin concentration was 0.6 (SD 0.8) g/l plasma when the extent of haemolysis (in four categories) was assessed with the naked eye (Hubbuch \& Schuckliess, 1989). To investigate the possibility that there had been haemolysis or that some other factor between venepuncture and centrifugation of the blood sample might have had an effect on the results, we conducted statistical analyses restricted, alternatively, to the thirty-six women (45\%) whose plasma samples showed no signs of haemolysis when inspected with the naked eye, or to the fifty-three women $(66 \%)$ whose blood samples had been in the mail for $1 \mathrm{~d}$ only.

\section{Results}

Table 1 presents the characteristics of the eighty study subjects according to soya-intake group. The mean ages were 35.7 years, 31.4 years, 31.5 years and 29.7 years respectively in the soya-intake groups 1, 2, 3 and 4 $(P<0.001$ for heterogeneity and trend). Thirty-one women were vegetarians and twelve were vegans; vegetarians were spread throughout the four soya-intake groups, whereas all vegans were in groups 3 and 4 .

Mean soya milk consumption showed an increase from group 1 to group 4 according to the estimates from both the FFQ and the food diary, but the FFQ-based estimates were substantially higher than the diary-based estimates in groups 3 and 4. For solid soya foods, the FFQ-based estimates were 4.5-fold higher than the diary-based estimates in group 2, whereas the diary-based estimates for solid soya foods were larger than the FFQ-based estimates in both groups 3 and 4 . Contrary to our expectation, the diary-based estimates for solid soya food consumption increased monotonically from group 1 to group 4 . The estimates of total daily isoflavone intakes (mg) were $0 \cdot 2,20 \cdot 0,17 \cdot 1$ and $48 \cdot 9$ based on the FFQ, and $0 \cdot 6,5 \cdot 9,19 \cdot 6$ and $33 \cdot 7$ based on the $7 \mathrm{~d}$ food diaries for group 1-4 respectively. Thus, the FFQ data indicated that the dietary isoflavone intakes for groups 2 and 3 would be very similar, whereas the food-diary data pointed to isoflavone intakes increasing steadily from group 1 to group 4.

The geometric mean concentrations of plasma isoflavones in the group with the highest soya intake (group 4) were more than 25 -fold higher than the mean concentrations in the group with the lowest soya intake (group 1), the plasma concentrations increasing steadily from group 1 to group 4 (Table 2). The geometric mean values for plasma

Table 1. Selected characteristics and soya-related dietary intakes of eighty premenopausal British women grouped according to soya intake*

(Values are means and standard deviations for twenty women per group)

\begin{tabular}{|c|c|c|c|c|c|c|c|c|}
\hline \multirow{2}{*}{$\begin{array}{l}\text { Soya-intake group } \\
\text { Variable }\end{array}$} & \multicolumn{2}{|c|}{1} & \multicolumn{2}{|c|}{2} & \multicolumn{2}{|c|}{3} & \multicolumn{2}{|c|}{4} \\
\hline & Mean & SD & Mean & SD & Mean & SD & Mean & SD \\
\hline Age (years) & $35 \cdot 7$ & $3 \cdot 3$ & $31 \cdot 4$ & 4.5 & $31 \cdot 5$ & $4 \cdot 4$ & $29 \cdot 7$ & $4 \cdot 2$ \\
\hline BMI $\left(\mathrm{kg} / \mathrm{m}^{2}\right)$ & $22 \cdot 8$ & 2.6 & $23 \cdot 8$ & 4.4 & $21 \cdot 8$ & $2 \cdot 5$ & $22 \cdot 4$ & 3.4 \\
\hline \multicolumn{9}{|l|}{ Soya foods $(\mathrm{g} / \mathrm{d})$} \\
\hline \multicolumn{9}{|l|}{ Soya milk } \\
\hline FFQ & 0 & 0 & 0 & 0 & 147 & 0 & 374 & 101 \\
\hline Food diary & 0 & 0 & $15 \cdot 2$ & $52 \cdot 1$ & 114 & 102 & 175 & $86 \cdot 2$ \\
\hline \multicolumn{9}{|l|}{ Solid soya foods } \\
\hline FFQ & 0.9 & 0 & $76 \cdot 5$ & $9 \cdot 1$ & $11 \cdot 6$ & $3 \cdot 3$ & $49 \cdot 2$ & $17 \cdot 1$ \\
\hline Food diary & $2 \cdot 8$ & $6 \cdot 3$ & $21 \cdot 1$ & $32 \cdot 0$ & $38 \cdot 2$ & $43 \cdot 4$ & 65.5 & $50 \cdot 3$ \\
\hline \multicolumn{9}{|l|}{ Total soya intake } \\
\hline FFQ & 0.9 & 0 & $76 \cdot 5$ & $9 \cdot 1$ & 158 & $3 \cdot 3$ & 424 & $98 \cdot 2$ \\
\hline Food diary & $2 \cdot 8$ & $6 \cdot 3$ & $36 \cdot 3$ & $83 \cdot 0$ & 153 & 117 & 240 & 101 \\
\hline \multicolumn{9}{|c|}{ Total isoflavones $(\mathrm{mg} / \mathrm{d})$} \\
\hline \multicolumn{9}{|l|}{ Soya milk } \\
\hline FFQ & 0 & 0 & 0 & 0 & $14 \cdot 1$ & 0 & $36 \cdot 1$ & $9 \cdot 7$ \\
\hline Food diary & 0 & 0 & 1.5 & $5 \cdot 0$ & $11 \cdot 0$ & $9 \cdot 9$ & $16 \cdot 9$ & $8 \cdot 3$ \\
\hline \multicolumn{9}{|l|}{ Solid soya foods } \\
\hline FFQ & 0.2 & 0 & $20 \cdot 0$ & $2 \cdot 3$ & $3 \cdot 0$ & 0.8 & $12 \cdot 9$ & 4.5 \\
\hline Food diary & 0.6 & 1.6 & $4 \cdot 4$ & $4 \cdot 6$ & 8.5 & $11 \cdot 4$ & $16 \cdot 9$ & 11.9 \\
\hline \multicolumn{9}{|l|}{ Total soya intake } \\
\hline FFQ & 0.2 & 0 & $20 \cdot 0$ & $2 \cdot 3$ & $17 \cdot 1$ & $0 \cdot 8$ & $48 \cdot 9$ & $9 \cdot 7$ \\
\hline Food diary & 0.6 & 1.6 & $5 \cdot 9$ & 8.5 & $19 \cdot 6$ & $15 \cdot 8$ & $33 \cdot 7$ & $15 \cdot 6$ \\
\hline
\end{tabular}

FFQ, food-frequency questionnaire.

* The soya-intake groups were based on data from FFQ as follows: group 1, no use of soya milk, solid soya foods less than once per month; group 2, no use of soya milk, solid soya foods five to six times per week or more often; group 3, $142 \mathrm{ml}$ soya milk daily, solid soya foods once per week or less; group $4, \geq 284 \mathrm{ml}$ soya milk daily, solid soya foods two to four times per week or more often. 
Table 2. Plasma genistein and daidzein concentrations in eighty premenopausal British women grouped according to soya intake*

(Values are geometric and arithmetic means and $95 \%$ confidence intervals for twenty women per group)

\begin{tabular}{cccccc}
\hline & \multicolumn{2}{c}{$\begin{array}{c}\text { Plasma daidzein } \\
(\mathrm{nmol} / \mathrm{l})\end{array}$} & & \multicolumn{2}{c}{$\begin{array}{c}\text { Plasma genistein } \\
(\mathrm{nmol} / \mathrm{l})\end{array}$} \\
\cline { 2 - 3 } \cline { 5 - 6 } Soya-intake group & Mean & $95 \% \mathrm{Cl}$ & & Mean & $95 \% \mathrm{Cl}$ \\
\hline Geometric mean concentrations & & & \\
1 & $4 \cdot 93$ & $2 \cdot 80,8 \cdot 66$ & & $14 \cdot 3$ & $7 \cdot 42,27 \cdot 4$ \\
2 & $8 \cdot 37$ & $4 \cdot 76,14 \cdot 7$ & & $16 \cdot 5$ & $8 \cdot 57,31 \cdot 6$ \\
3 & $39 \cdot 2$ & $22 \cdot 3,69 \cdot 0$ & & 119 & $61 \cdot 8,228$ \\
4 & 132 & $75 \cdot 4,233$ & & 378 & 197,726 \\
Arithmetic mean concentrations & & & & \\
1 & $8 \cdot 05$ & $0,51 \cdot 2$ & & $27 \cdot 8$ & 0,163 \\
2 & $23 \cdot 7$ & $0,66 \cdot 9$ & & $58 \cdot 8$ & 0,194 \\
3 & $94 \cdot 2$ & $51 \cdot 0,137$ & & 285 & 149,420 \\
4 & 178 & 135,221 & & 525 & 390,661 \\
\hline
\end{tabular}

* The soya-intake groups were based on data from food-frequency questionnaires as follows: group 1, no use of soya milk, solid soya foods less than once per month; group 2, no use of soya milk, solid soya foods five to six times per week or more often; group $3,142 \mathrm{ml}$ soya milk daily, solid soya foods once per week or less; group $4 \geq 284 \mathrm{ml}$ soya milk daily, solid soya foods two to four times per week or more often. For details of experimental procedures, see p. 416.

daidzein (nmol/1) were 4.9, 8·4, $39 \cdot 2$ and 132 for groups $1-4$ respectively $(P 0.0001$ for heterogeneity); the corresponding values for plasma genistein $(\mathrm{nmol} / \mathrm{l})$ were $14.3,16.5$, 119 and 378 ( $P$ 0.0001 for heterogeneity). Adjustment for age slightly decreased the difference between the groups with the highest and the lowest soya intake (groups 1 and 4, but the geometric mean concentrations of plasma isoflavones remained more than 20-fold higher in the group with the highest soya intake highest compared with those of the group with the lowest soya intake. The arithmetic means showed the same pattern of variation as the geometric means, but the arithmetic mean values were much higher than the geometric mean values because of the strongly positively skewed distributions of isoflavone concentrations.

The Spearman correlation coefficient between plasma daidzein and plasma genistein was very high ( $r$ 0.95). All diary-based estimates of dietary soya intake showed very high correlations with plasma daidzein and genistein concentrations (dietary total soya and isoflavone intakes $r$ 0.78-0.80, soya milk intake $r 0 \cdot 74-0 \cdot 77$, solid soya food intake $r$ 0.66-0.69; Table 3; $P<0.001)$. High correlations with plasma daidzein and genistein were observed for total soya and soya milk intakes from the FFQ-based estimates $(r 0.72-0.74, P<0.001)$, and also for daidzein and genistein intakes $(r$ 0.69-0.74, $P<0.001)$. The FFQ-based estimate for total isoflavone intake also showed a reasonably high correlation with plasma isoflavones $(r 0.53-0.56, P<0.001)$, but the correlations for FFQ-based estimates of solid soya food intakes with plasma isoflavones were clearly weaker ( $r \quad 0 \cdot 24-0.28)$ but remained statistically significant
Table 3. Correlation (Spearman correlation coefficients) between dietary intakes of soya foods and isoflavones, based on food-frequency questionnaires (FFQ) and $7 d$ food diaries, and plasma concentrations of daidzein and genistein in eighty premenopausal British women*

\begin{tabular}{|c|c|c|}
\hline Dietary intake & Plasma daidzein & Plasma genistein \\
\hline \multicolumn{3}{|l|}{ Soya milk (g/d) } \\
\hline FFQ & 0.72 & 0.72 \\
\hline Food diary & 0.74 & 0.77 \\
\hline \multicolumn{3}{|c|}{ Solid soya foods $(\mathrm{g} / \mathrm{d})$} \\
\hline FFQ & $0.28 \dagger$ & $0.24 \dagger$ \\
\hline Food diary & 0.69 & 0.66 \\
\hline \multicolumn{3}{|l|}{ Total soya $(\mathrm{g} / \mathrm{d})$} \\
\hline $\mathrm{FFQ}$ & 0.74 & 0.73 \\
\hline Food diary & 0.78 & 0.78 \\
\hline \multicolumn{3}{|l|}{ Daidzein (mg/d) } \\
\hline FFQ & 0.74 & 0.71 \\
\hline Food diary & 0.79 & 0.80 \\
\hline \multicolumn{3}{|c|}{ Genistein (mg/d) } \\
\hline FFQ & 0.72 & 0.69 \\
\hline Food diary & 0.79 & 0.80 \\
\hline \multicolumn{3}{|c|}{ Total isoflavones $(\mathrm{mg} / \mathrm{d})$} \\
\hline FFQ & 0.56 & 0.53 \\
\hline Food diary & 0.79 & 0.80 \\
\hline
\end{tabular}

* All correlations, except those indicated, were highly significant (two-sided, $P<0.001$ ).

† Correlation between FFQ-based estimate and plasma daidzein was significant at $P=0.012$, and that for FFQ-based estimate and plasma genistein was significant at $P=0.034$

( $P=0.012$ for plasma daidzein, $P=0.034$ for plasma genistein). No differences were observed between the correlation coefficients for plasma daidzein compared with those for plasma genistein.

Restriction of the statistical analyses to the thirty-six women whose blood samples showed no signs of haemolysis when inspected with the naked eye, or to the fifty-three women whose blood samples had been in the mail for only $1 \mathrm{~d}$, did not materially change the mean concentrations of isoflavones nor the correlation coefficients compared to with the values shown in Tables 2 and 3.

\section{Discussion}

Reported soya intakes varied more than 100-fold between the four intake groups. For subjects in groups 3 and 4, soya milk intakes estimated from the FFQ data were much higher than those estimated from the diary data. This finding that the FFQ yields higher average daily intakes than other methods has been noted before, and probably represents an overestimation of milk intake by the FFQ (Bingham et al. 1997). Despite this finding, the correlation coefficients between dietary soya milk intakes and plasma isoflavone concentrations were high, implying that the measurement error in the absolute levels has little effect on the relative validity of FFQ-based soya milk assessment. Likewise, any haemolysis-related effects would have been too small to have an important effect on the results of the present study.

Fig. 1. Studies on plasma or serum daidzein concentrations in adults *Concentrations are given as geometric means; all other concentrations are arithmetic means. For the present study, eighty premenopausal women were allocated to groups according to soya intake; groups $1-4$ represent increasing soya intake, with twenty women per group (For details, see Table 1 and p. 416). 


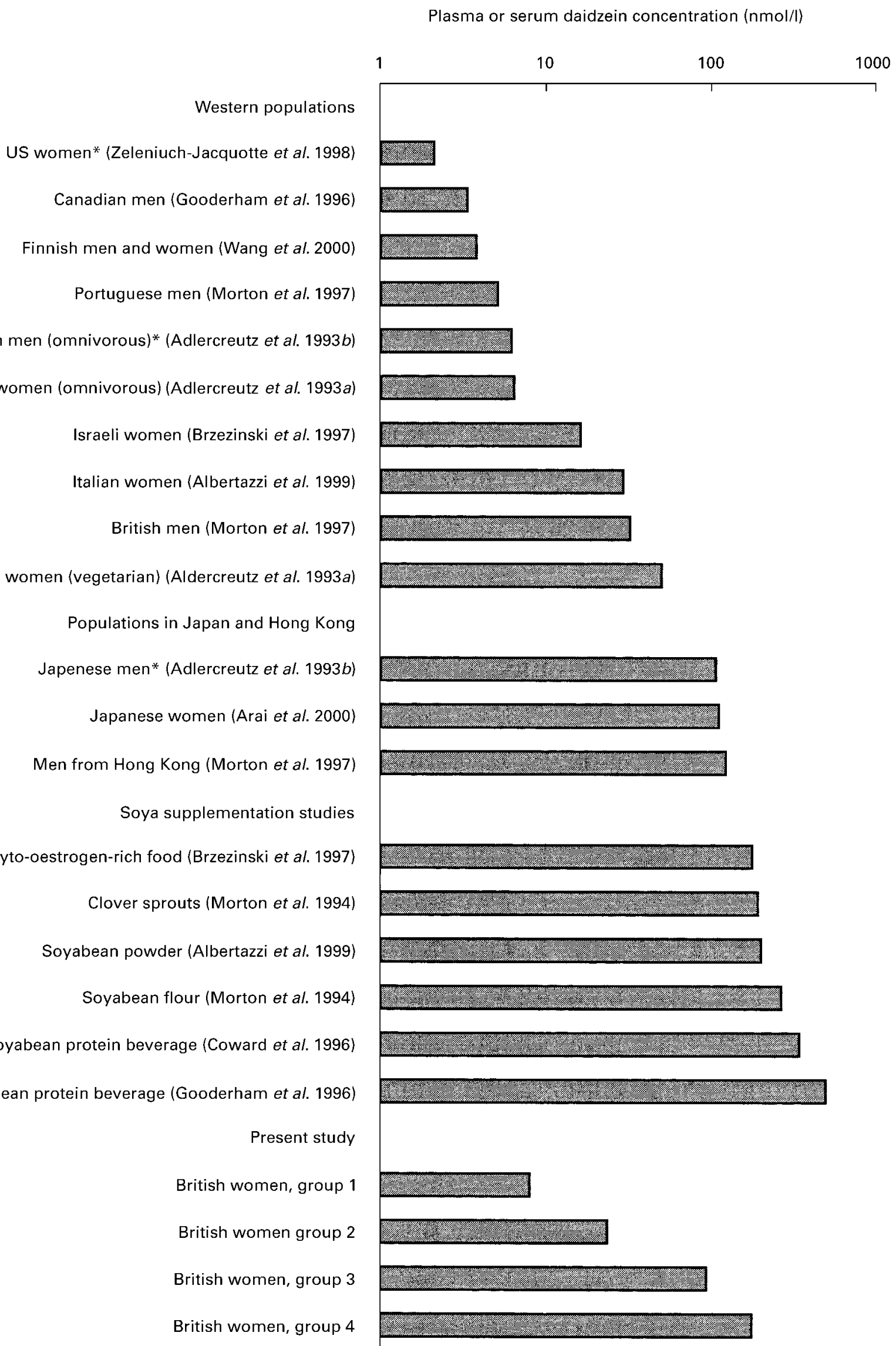

Finnish women (vegetarian) (Aldercreutz et al. 1993a)

Populations in Japan and Hong Kong

Finnish women (omnivorous) (Adlercreutz et al. 1993a)

(Arali women (Brzezinski et al. 1997)

Italian women (Albertazzi et al. 1999)

British men (Morton et al. 1997)

Japenese men* (Adlercreutz et al. 1993b)

Japanese women (Arai et al. 2000)

Men from Hong Kong (Morton et al. 1997)

Soya supplementation studies

Phyto-oestrogen-rich food (Brzezinski et al. 1997)

Clover sprouts (Morton et al. 1994)

Soyabean powder (Albertazzi et al. 1999)

Soyabean flour (Morton et al. 1994)

Soyabean protein beverage (Coward et al. 1996)

Soyabean protein beverage (Gooderham et al. 1996)

Present study

British women, group 1

British women group 2

British women, group 3

British women, group 4 
Based on the FFQ data, we expected that the consumption of solid soya foods would be highest in group 2 (where solid soya foods were reportedly consumed five to six times per week, or more often) and second highest in group 4 (where solid soya foods were reportedly consumed two to four times per week or more often), but the diary data showed that consumption of solid soya foods increases steadily from group 1 to group 4 . This discrepancy points to a relatively poor performance of the FFQ question in relation to the question on solid soya foods. The finding may be partly a result of the lack of specificity of the question, i.e. that it covered the intakes of several foods (including tofu, soya meat, textured vegetable protein and vegeburgers), and partly a result of a significant part of the ingested soyabean being obtained from sources unrecognized by the FFQ.

We observed correlation coefficients from 0.65 to 0.75 between dietary soya food and isoflavone intakes and plasma isoflavones. These values are higher than the correlations between diary-based dietary isoflavone intakes and plasma isoflavones in a recent Japanese study (energyadjusted Pearson correlations from 0.33 to $0.42, P<0.001$; Arai et al. 2000). They are also higher than correlations for most other nutrients assessed with the EPIC-FFQ (median Spearman correlation for eighteen nutrients 0.55 ) and broadly similar to the correlations observed for most other nutrients assessed with the EPIC food diary (median Spearman correlation 0.72; Bingham et al. 1997). It appears that our non-random subject selection has contributed, particularly in the case of the FFQ, to the high levels of correlation coefficients observed in the present study by increasing the standard deviation of dietary isoflavone measurements. However, non-random subject selection would not create a relationship where none exists; i.e. if there were no real association between dietary and plasma isoflavones, then our non-random subject selection would not produce an association.

The correlation coefficients between estimated dietary isoflavone intakes and plasma isoflavones were not higher than the respective correlation coefficients between total soya intakes and plasma isoflavones (in fact, the FFQ-based estimate for total isoflavones showed weaker correlations with plasma isoflavones than the FFQ-based estimate for total soya food intake). Thus, it appears that published data on measurements of isoflavones in soya products did not add to the information provided by dietary soya intake alone. This may be because of the large geographical and temporal variations in isoflavone contents in different foods; in consequence, the isoflavone contents published in any particular food table cannot be expected to provide accurate estimates of the isoflavone content of the same food studied in a different time and place.

Both the FFQ and the diary provided estimates for soya intake which correlated well with plasma isoflavone levels. The diary gave a more consistent response amongst the four exposure groups than the FFQ and was a tool that could be used with somewhat greater confidence; however, coding the diaries is much more time consuming and expensive than coding the FFQ. For many epidemiological purposes, the two questions on soya milk consumption in the FFQ should give reasonably valid estimates for isoflavone consumption.
The dietary intake levels of isoflavones in women who consumed soya as a part of their regular diet in the present study were close to the levels in Japanese adults consuming a traditional diet, which has recently been reported to provide <30 mg isoflavones/d (Messina, 1995; Watanabe et al. 2000). Fig. 1 summarizes the studies on daidzein concentrations in human serum or plasma (Adlercreutz et al. 1993a,b; Morton et al. 1994, 1997; Coward et al. 1996; Gooderham et al. 1996; Brzezinski et al. 1997; ZeleniuchJacquotte et al. 1998; Albertazzi et al. 1999; Arai et al. 2000; Wang et al. 2000; Watanabe et al. 2000). In the present study the plasma isoflavone concentrations in British women who used little or no soya were as low as those previously observed in typical Western populations (Adlercreutz et al. 1993a,b; Gooderham et al. 1996; Morton et al. 1997; Zeleniuch-Jacquotte et al. 1998; Wang et al. 2000); the only exceptions are an international study that reported surprisingly high daidzein concentrations in British men but not in Portuguese men (Morton et al. 1997), and perhaps two soya supplementation studies that observed equally high concentrations at baseline in omnivorous Israeli women (Brzezinski et al. 1997) and Italian women (Albertazzi et al. 1999). The concentrations in the group with the second-highest soya intake in the current study were similar to the levels that have been reported previously for vegetarian Finnish women (Adlercreutz et al. 1993a); the concentrations in the group with the highest soya intake were of the same magnitude as the concentrations in Japanese men or women consuming traditional diets (Adlercreutz et al. 1993b; Morton et al. 1997). However, the plasma isoflavone concentrations after supplementation of regular diets with soya appear to be even higher (Morton et al. 1994; Coward et al. 1996; Gooderham et al. 1996; Brzezinski et al. 1997; Albertazzi et al. 1999).

In conclusion, the present study suggests that, in a Western population, dietary intakes of soya isoflavones can be estimated reliably enough for epidemiological purposes using either a FFQ or a $7 \mathrm{~d}$ food diary. The food diaries performed somewhat better than the FFQ, where the question related to the consumption of solid soya foods did not add much to the information obtained by the questions related to soya milk consumption only. Alternatively, for prospective studies with no questionnaire or diary data on soya intake, plasma values of daidzein or genistein could be used as biomarkers of soya intake. In some British women consuming soya foods as a part of their regular diet, the plasma concentrations of isoflavones were close to the concentrations in Asian populations.

\section{Acknowledgements}

We thank all the participants of this study and the EPIC study staff at the Cancer Epidemiology Unit, Imperial Cancer Research Fund, Oxford, especially Ms Jennie Postans who coded the food diaries for soya consumption. P. V. was supported by the European Commission Marie Curie Fellowship BMH4-CT98-5113 under the Biomedicine and Health Programme, the Academy of Finland grant no. 42022, the Finnish Cancer Foundations and the Finnish Medical Society Duodecim. H.A. has been supported by the Sigrid Juselius Foundation. The EPIC study is supported by 
the Imperial Cancer Research Fund and by the European Commission under the Europe Against Cancer Programme.

\section{References}

Adlercreutz H, Fotsis T, Lampe J, Wähälä K, Mäkelä T, Brunow G \& Hase T (1993a) Quantitative determination of lignans and isoflavonoids in plasma of omnivorous and vegetarian women by isotope dilution gas chromatography-mass spectrometry. Scandinavian Journal of Clinical and Laboratory Investigations 53, Suppl. 215, 5-18.

Adlercreutz H, Markkanen H \& Watanabe S (1993b) Plasma concentrations of phyto-oestrogens in Japanese men. Lancet 342, 1209-1210.

Adlercreutz H \& Mazur W (1997) Phyto-oestrogens and Western diseases. Annals of Medicine 29, 95-120.

Albertazzi P, Pansini F, Bottazzi M, Bonaccorsi G, De Aloysio D \& Morton MS (1999) Dietary soy supplementation and phytoestrogen levels. Obstetrics \& Gynecology 94, 229-231.

Arai Y, Uehara M, Sato Y, Kimira M, Eboshida A, Adlercreutz H $\&$ Watanabe S (2000) Comparison of isoflavones among dietary intake, plasma concentrations and urinary excretion for accurate estimation of phytoestrogen intake. Journal of Epidemiology $\mathbf{1 0}$, 127-135.

Bingham SA, Atkinson C, Liggins J, Bluck L \& Coward A (1998) Phyto-oestrogens: where are we now? British Journal of Nutrition 79, 393-406.

Bingham SA, Gill C, Welch A, Cassidy A, Runswick SA, Oakes S, Lubin R, Thurnham DI, Key TJA, Roe L, Khaw K-T \& Day NE (1997) Validation of dietary assessment methods in the UK arm of EPIC using weighed records, and 24-hour urinary nitrogen and potassium and serum vitamin $\mathrm{C}$ and carotenoids as biomarkers. International Journal of Epidemiology 26, Suppl. 1, 137-151.

Brzezinski A, Adlercreutz H, Shaoul R, Rösler A, Shmueli A, Tanos V \& Schenker JG (1997) Short-term effects of phytoestrogen-rich diet on postmenopausal women. Menopause 4, 89-94.

Coward L, Kirk M, Albin N \& Barnes S (1996) Analysis of plasma isoflavones by reversed-phase HPLC-multiple reaction monitoring-mass spectrometry. Clinica Chimica Acta 121, 121-142.

Fournier DB, Erdman JWJ \& Gordon GB (1998) Soy, its components, and cancer prevention: a review of the in vitro, animal, and human data. Cancer Epidemiology, Biomarkers \& Prevention 7, 1055-1065.

Gooderham MH, Adlercreutz H, Ojala ST, Wähälä K \& Holub BJ (1996) A soy protein isolate rich in genistein and daidzein and its effects on plasma isoflavone concentrations, platelet aggregation, blood lipids and fatty acid composition of plasma phospholipid in normal men. Journal of Nutrition 126, 2000-2006.

Greenstein J, Kushi L, Zheng W, Fee R, Campbell D, Sellers T \& Folsom A (1996) Risk of breast cancer associated with intake of specific foods and food groups. American Journal of Epidemiology 143, Suppl., 36.

Hubbuch A \& Schuckliess F (1989) Quality Assurance. Interference by Haemolysis, Mannheim, Germany: Boehringer Mannheim.

International Agency for Research on Cancer (2000). International Agency for Research on Cancer Database. http://www.dep.iarc. fr/dataava/dataicon.htm

Jacobsen BK, Knutsen SF \& Fraser GE (1998) Does high soy milk intake reduce prostate cancer incidence? The Adventist Health Study (United States). Cancer Causes and Control 9, 553-557.

Key TJ, Sharp GB, Appleby PN, Beral V, Goodman MT, Soda M \& Mabuchi K (1999) Soya foods and breast cancer risk: a prospective study in Hiroshima and Nagasaki, Japan. British Journal of Cancer 81, 1248-1256.

Messina M (1995) Isoflavone intakes by Japanese were overestimated. American Journal of Clinical Nutrition 62, 645.

Morton MS, Chan PSF, Cheng C, Blacklock N, MatosFerreira A, AbranchesMonteiro L, Correia R, Lloyd S \& Griffiths K (1997) Lignans and isoflavonoids in plasma and prostatic fluid in men: samples from Portugal, Hong Kong, and the United Kingdom. Prostate 32, 122-128.

Morton MS, Wilcox G, Wahlqvist ML \& Griffiths K (1994) Determination of lignans and isoflavonoids in human female plasma following dietary supplementation. Journal of Endocrinology 142, 251-259.

Setchell KDR (1998) Phytoestrogens: the biochemistry, physiology, and implications for human health of soy isoflavones. American Journal of Clinical Nutrition 68, Suppl., 1333-1346.

US Department of Agriculture-Iowa State University (1999). Database on Isoflavone Content of Foods http://warp.nal.usda. gov/fnic/foodcomp/Data/isoflav/isoflav.html

Wang GJ, Lapcik O, Hampl R, Uehara M, Al-Maharik N, Stumpf K, Mikola H, Wähälä K \& Adlercreutz H (2000) Time-resolved fluoroimmunoassay of plasma daidzein and genistein. Steroids 65, 339-348.

Watanabe S, Yusuke A, Haba R, Uehara M, Adlercreutz H, Shimoi $\mathrm{K}$ \& Kinae N (2000) Dietary intake of flavonoids and isoflavonoids by Japanese and their pharmacokinetics and bioactivities. In Phytochemicals and Phytopharmaceuticals, pp. 164-174 [F Shahidi and C-T Ho, editors]. Champaign, IL: AOCS Press.

Zeleniuch-Jacquotte A, Adlercreutz H, Akhmedkhanov A \& Toniolo P (1998) Reliability of serum measurements of lignans and isoflavonoid phytoestrogens over a two-year period. Cancer Epidemiology, Biomarkers and Prevention 7, 885-889. 